\title{
HISTOPATOLOGÍA DE LAS LESIONES ATEROMATOSAS \\ Estudio en muestras obtenidas de hombres fallecidos por causa no cardíaca
}

Aída Romero S., Jaydi Acosta A., Nelcy Pineda G.*

\section{Resumen}

El objeto de esta investigación es validar el sistema aterométrico, método morfométrico que permite la identificación y medición de las lesiones ateromatosas precursoras de la ateroesclerosis, y cotejarlo con la microscopía óptica, en 100 arterias aortas sin ninguna lesión traumática pertenecientes a hombres entre 15 y 60 años, quienes fallecieron por causa no cardíaca e ingresaron al Instituto Nacional de Medicina Legal de Bogotá entre junio y diciembre de 2000.

\section{Introducción}

El sistema aterométrico, método desarrollado y puesto en práctica por el centro de investigación y referencia de ateroesclerosis de la Habana (Cuba), brinda una herramienta útil para la evaluación cualitativa y cuantitativa de la lesión ateroesclerótica.

La ateroesclerosis (AE) es la causa principal de las enfermedades cardiovasculares y estas, a su vez, constituyen la primera causa de muerte no traumática de la población colombiana. La palabra ateroesclerosis fue introducida por Lobstein en 1833 y evolucionó como termino general en la designación de la enfermedad arterial. ${ }^{1}$

En la actualidad puede interpretarse como la respuesta defensiva obligada del tejido conectivo de la pared arterial ante agresiones permanentes; es un evento crónico, silencioso, generalmente ignorado y desconocido. Es frecuente en pacientes de sexo masculino y raza negra, aumenta con la edad y se asocia a factores de riesgo específicos como la obesidad, hipertensión arterial, ingesta de lípidos, vida sedentaria, diabetes y hábito de fumar. ${ }^{2}$

* Citohistotecnólogas, Fundación Universitaria de Ciencias de la Salud. Bogotá, D.C., Colombia
La AE es la forma más común de ateroesclerosis, caracterizada desde el punto de vista anatómico por la presencia anormal de material lipídico en la túnica íntima de la arteria, lo cual disminuye la elasticidad, debilita la pared y permite el depósito de sustancias con la consiguiente disminución del calibre de la luz; esto trae como consecuencia la reducción del flujo sanguíneo y la formación de trombos y aneurismas. Ante el estímulo agresor el organismo puede responder mediante inflamación, inmunidad y reparación, procesos que conllevan a la producción de una cicatriz que culminará como lesión ateroesclerótica; ésta se desarrolla en la túnica íntima de las arterias musculares y elásticas como resultado de dos procesos: acumulación de lípidos y proliferación del músculo liso en la íntima

Los factores envueltos en la formación de la placa ateroesclerótica son:

a) Endotelio vascular

b) Célula muscular lisa

c) Monocito-macrófago

d) Leucocito, linfocito y neutrófilo

e) Factores de coagulación. ${ }^{3}$ 
La primera lesión detectable es la estría grasa, caracterizada macroscópicamente por su aspecto levantado, color amarillo y orientación paralela con respecto al vaso. Es delgada y contiene cúmulos de lípidos en la íntima y se encuentra tanto en jóvenes como en adultos.

La lesión característica de la AE es la placa fibrosa que contiene dos componentes morfológicos: capa fibrosa y ateroma. La primera está formada por tejido conectivo fibroso, macrófagos y células musculares lisas. Desde el punto de vista macroscópico se caracteriza por una placa dura y blanquecina, elevada, a veces gris pálida o blanca nacarada y de consistencia firme con una superficie que protruye hacia la luz.

El ateroma se refiere al acúmulo de grasa pero no se usa para designar toda afección, ya que se trata de los depósitos de lípidos que forman la parte media de la lesión característica de la AE. A medida que la acumulación y proliferación de células progresa, la placa aumenta de tamaño hasta obstruir la luz; al microscopio se encuentran macrófagos, linfocitos, fibroblastos, colágeno y células musculares lisas que rodean el centro de la lesión compuesto por células espumosas en degeneración, detritus celulares, macrófagos, eritrocitos, plaquetas, cristales de colesterol y depósitos ocasionales de calcio.

Los factores que contribuyen a la progresión de la lesión simple a complicada son: citoquinas, linfocitos T, endotelio y trombosis. En fases avanzadas aumenta la necrosis, la celularidad y el depósito de lípidos, la placa se neovasculariza en forma progresiva hasta producir una ruptura en la íntima, lo cual causa trombosis aguda en la pared arterial. Los eventos críticos que caracterizan esta lesión son: trombosis en la placa fibrosa, neovascularizacion, adelgazamiento de la túnica media y calcificación. ${ }^{4}$

\section{Metodología}

Con el propósito de valorar el sistema aterométrico se realizó una investigación de tipo prospectivo, descriptivo y analítico en la que se recolectaron 100 arterias aortas sin lesión traumática de hombres en- tre 15-60 años fallecidos por causa no cardíaca en el Instituto Nacional de Medicina Legal (INML), en el periodo junio a diciembre del año 2000 con previa autorización del departamento de investigación científica del INML.

\section{Materiales y métodos}

Para la manipulación de especímenes se requieren tablas y cubetas de fijación, tijeras para cirugía oftálmica, bolsas plásticas, formol, acetatos, coloración especial de Sudán. Y cámara fotográfica digital

Para el proceso microscópico se usan alcoholes, xiloles, parafinas, coloraciones especiales como tricrómico de Van Gieson, elástica, pentacromática y la hematoxilina eosina, de rutina láminas cubreobjetos, resina y cámara fotográfica adaptada al microscopio.

\section{Técnica del sîstema aterométrico}

Es un conjunto de métodos y procedimientos patomorfológicos y morfométricos, que se utilizan para la caracterización de la lesión ateroesclerótica en cualquier arteria o sector vascular. Es un instrumento ágil de extrema confiabilidad que permite el conocimiento preciso de la enfermedad ateroesclerótica y sus consecuencias orgánicas en aspectos patomorfológicos, morfométricos, etiopatogénicos y epidemiológicos. Además unificó los conocimientos de los investigadores de $\mathrm{AE}$ en el mundo. ${ }^{2}$

\section{Método para la disección y preparación de la arteria aorta}

a. Separar la aorta de sus relaciones anatómicas habituales.

b. Aislarla en su parte proximal de la pulmonar y en la distal de las ilíacas.

c. Retirar el tejido adiposo de la adventicia.

d. Realizar un corte longitudinal desde la zona proximal hasta la distal por los surcos intercostales. 
e. Adherir la arteria por su adventicia sobre una tabla de fijación e identificar los especímenes con el número del protocolo y la fecha.

f. Fijar en formalina al $10 \%$.

\section{Coloración microscópica de las lesiones ateroscleróticas según la técnica de Holman}

La coloración propuesta por Holman facilita la visualización de las estrías o bandas adiposas y de los depósitos lipídicos subintimales alrededor de las placas ateroescleróticas, poco apreciables sin previa coloración, lo que permite una mejor clasificación y morfometría.

Los pasos a seguir son los siguientes: las arterias previamente fijadas en formalina y separadas de su cartón, son lavadas en agua corriente, luego se introducen en alcohol al $70 \%$ por 15 segundos, para pasarlas, luego, a la solución de Herxheimer durante 15 minutos, la cual está compuesta de $500 \mathrm{ml}$ de alcohol al 80\%, $500 \mathrm{ml}$ de acetona y $5 \mathrm{~g}$ de Sudán IV. Se mezcla y se filtra.

El recipiente a emplear debe estar bien tapado para evitar la evaporación de la solución y moverse frecuentemente con el fin de facilitar una coloración homogénea. La solución de Herxheimer puede utilizarse varias veces, siempre filtrándola antes y después de su uso.

Al finalizar esta coloración la íntima de las arterias queda coloreada de rojo y se llevan a alcohol al $80 \%$ durante 25 minutos, y un nuevo paso por alcohol al $80 \%$ durante otros 25 minutos, lo que permite la adecuada diferenciación de las lesiones y de la íntima. Las bandas adiposas y los acúmulos lipídicos se tiñen de rojo, mientras que la íntima normal recupera su coloración habitual. El alcohol empleado en este paso debe ser limpio, sin uso previo. Por último, se lavan las arterias en agua corriente durante una hora y quedan listas para someterlas al análisis cualitativo y cuantitativo del sistema aterométrico. ${ }^{2}$

\section{Evaluación cualitativa del proceso ateroesclerótico de las arterias}

Consiste en clasificar las alteraciones que forman parte del proceso ateroesclerótico, calcando lo más exacto posible la arteria en un acetato e identificando con diferentes colores cada una de las lesiones.

\section{Evaluación cuantitativa del proceso ateroesclerótico en las arterias}

Para este análisis se realizan grupos de medidas que constituirán la formación primaria a partir de la cual se obtiene el resto de las variables que conforman el sistema aterométrico, que se dividen en descriptivas y ponderativas. Las primeras describen el área total de la arteria, longitud del vaso, el área afectada por cualquier tipo de lesión y la libre de lesión, expresadas en milímetros cuadrados; las segundas permiten estimar la severidad del proceso mediante los siguientes índices:

a. De obstrucción: suma de las lesiones que protruyen hacia la luz arterial, como son la placa fibrosa y complicada;

b. De estenosis: es el espacio que queda libre para la circulación de la sangre y

c. De benignidad: área libre de AE.

\section{Técnica histológica que permite corroborar el sistema aterométrico}

Para llevar a cabo el procedimiento histológico se eligieron seis aortas (dos por cada una de las lesiones que macroscópicamente con la técnica de Holman se evidenciaron). Se realizaron cortes a diferentes niveles (toráxico, abdominal, tronco celiaco y bifurcación en iliacas) para estudiarlos en microscopía óptica.

Se llevó a cabo el proceso histológico usual de fijación, deshidratación, aclaramiento, inhibición, inclusión, corte (cuatro láminas), coloración (hematoxilina eosina de rutina y especiales de tricrómico de Masson, fibras elásticas y pentacromática) y montaje. ${ }^{5}$

La información cualitativa fue recolectada teniendo como base los protocolos de necropsia en el Instituto Nacional de Medicina Legal con datos de edad, talla, peso, ocupación, causa de muerte, alcohol y drogas. La información cuantitativa se obtuvo mediante el empleo del sistema aterométrico. 
Dentro de los métodos estadísticos utilizados para el análisis se mezclaron las siguientes variables: edad, índice de obstrucción, causa de muerte y sedentarismo.

\section{Resultados de la macroscopía con la microscopía}

En el análisis estadístico se emplearon gráficas de tendencia, que nos permiten correlacionar diferentes variables como índice de masa corporal (IMC), oficio, uso de drogas y alcohol; sin embargo, debido a la falta de información en los protocolos de autopsia se tomó la variable más completa y representativa del estudio (edad), la cual fue correlacionada con cada una de las lesiones características de la arterioesclerosis (Figuras 1-12).

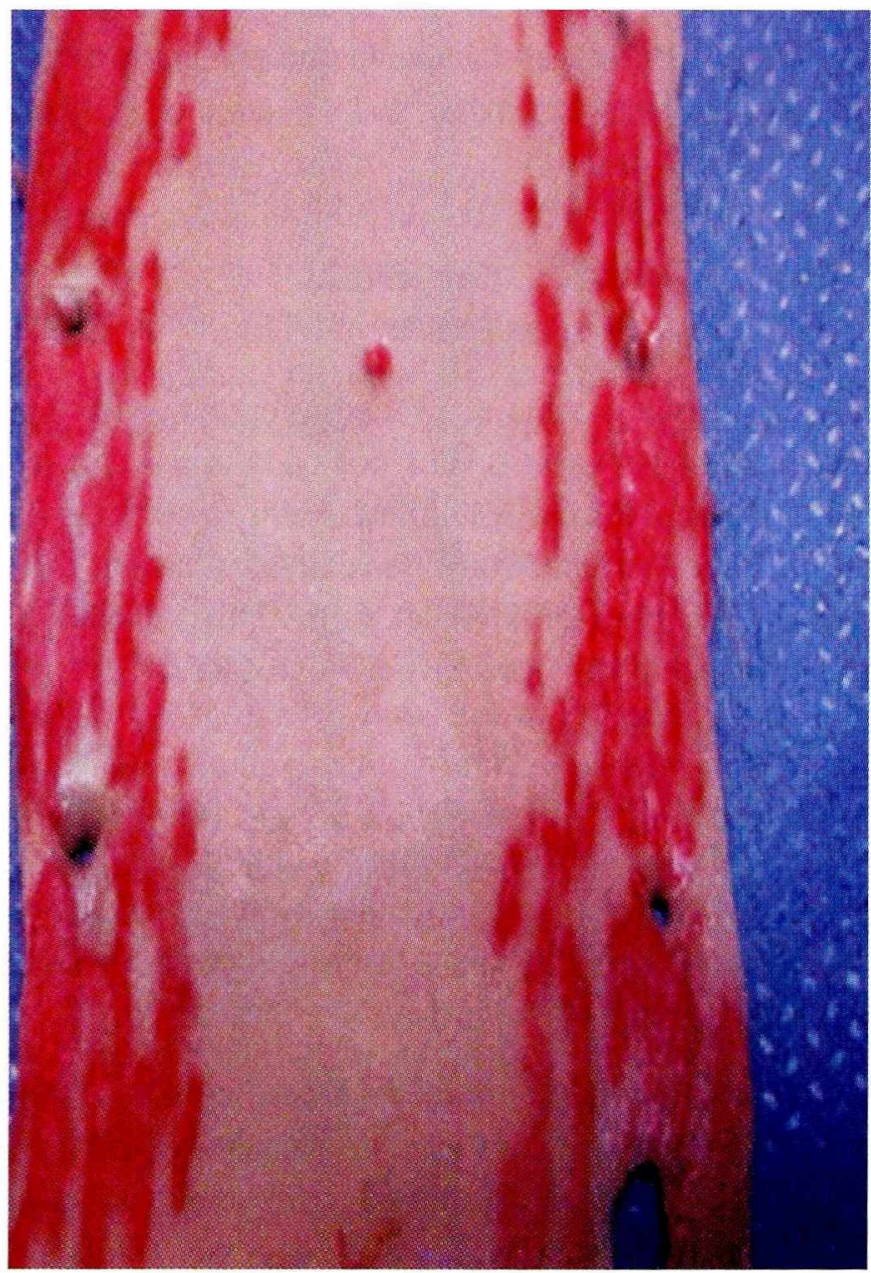

Figura 1. Estría adiposa con técnica de Holman (lesiones lineales sin ninguna elevación).

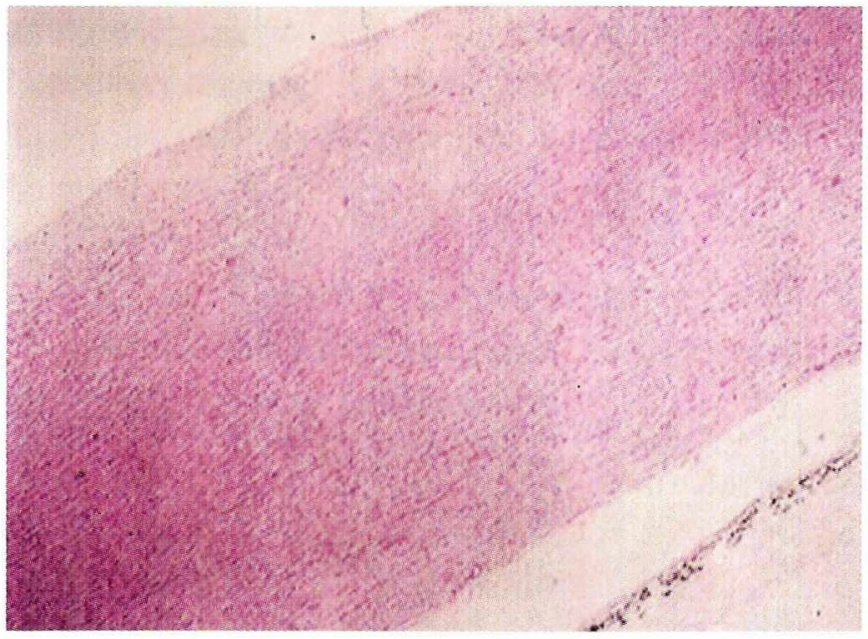

Figura 2. Coloración de HE, objetivo de 4x. La estructura conserva sus tres capas.

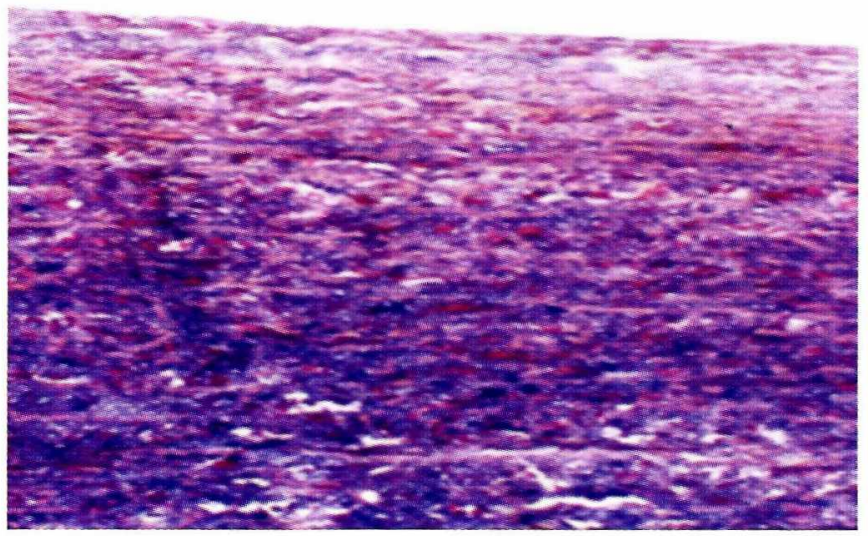

Figura 3. Coloración de tricrómico en objetivo de 10x. Se identifican fibras colágenas.

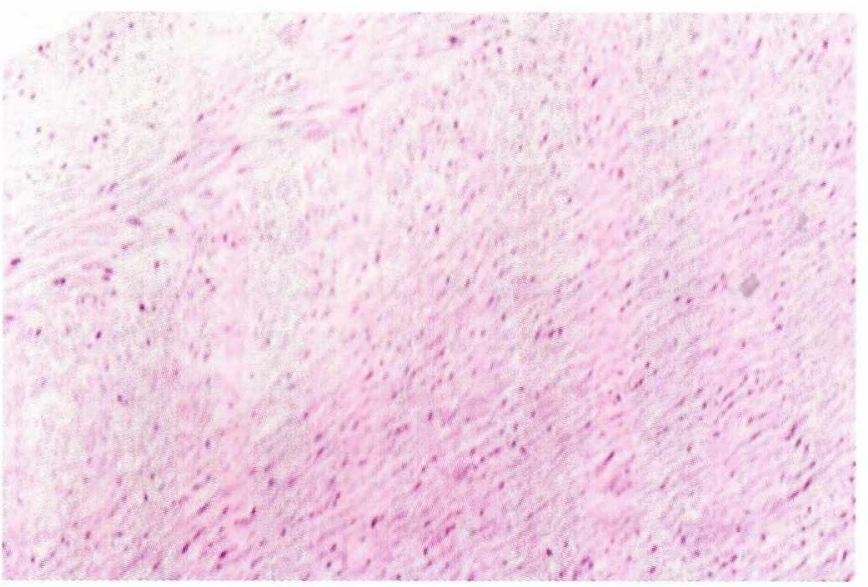

Figura 4. Coloración de HE, con objetivo de 40x. Se observa con más detalle la proliferación de macrófagos y el acúmulo de lípidos en la íntima. 


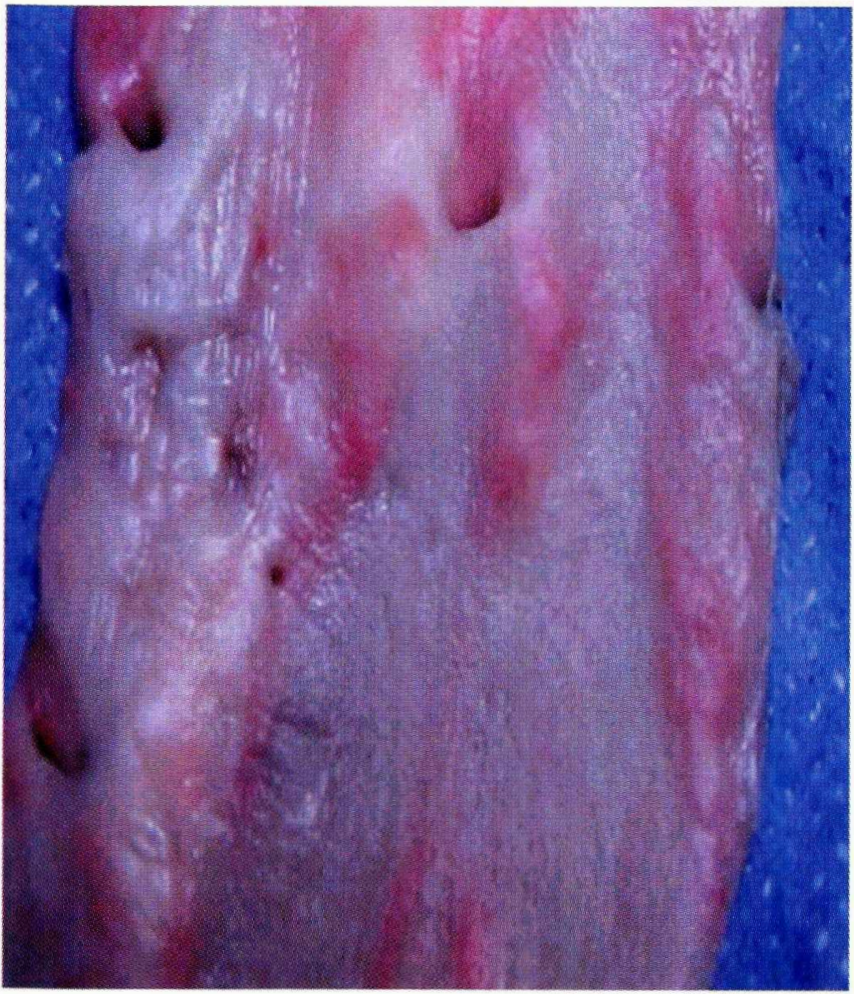

Figura 5. Placa fibrosa con técnica de Holman: zonas sobresalientes, palpables, de consistencia firme y color blanco nacarado.

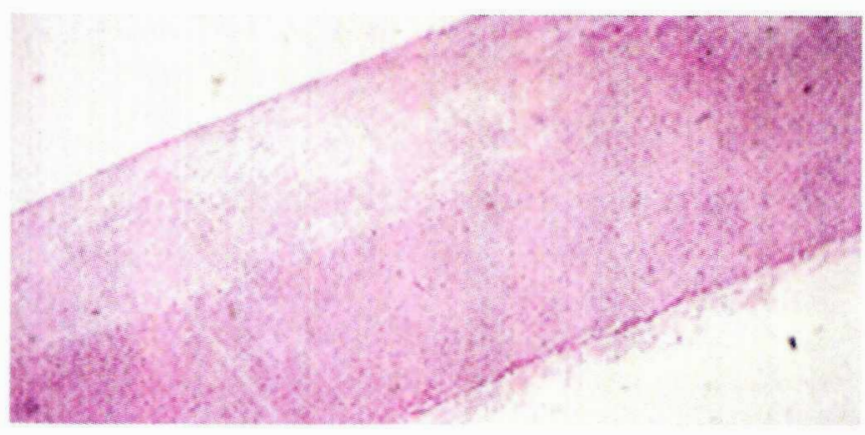

Figura 6. Coloración de $\mathrm{HE}$, con objetivo de $4 x$. La estructura se encuentra alterada en la capa media.

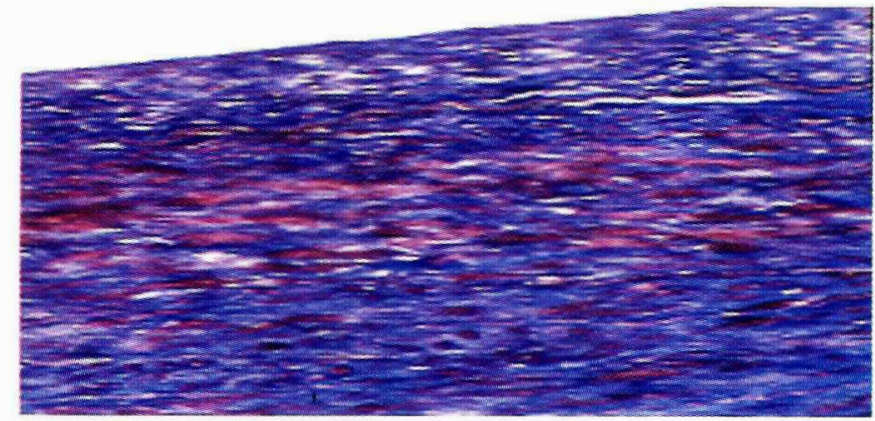

Figura 7. Coloración de tricrómico con objetivo de $10 x$. Se identifican menos fibras colágenas y fibras musculares.

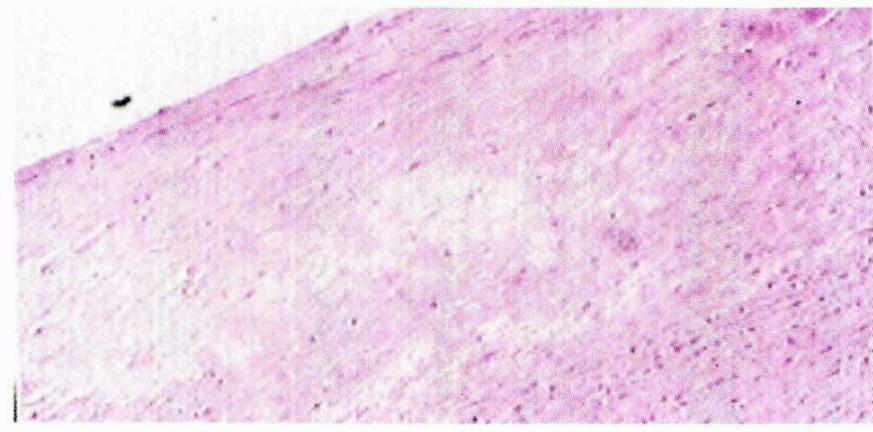

Figura 8. Coloración de HE con objetivo de 40x. Se observa con más detalle la proliferación de macrófagos y fibroblastos en la íntima.

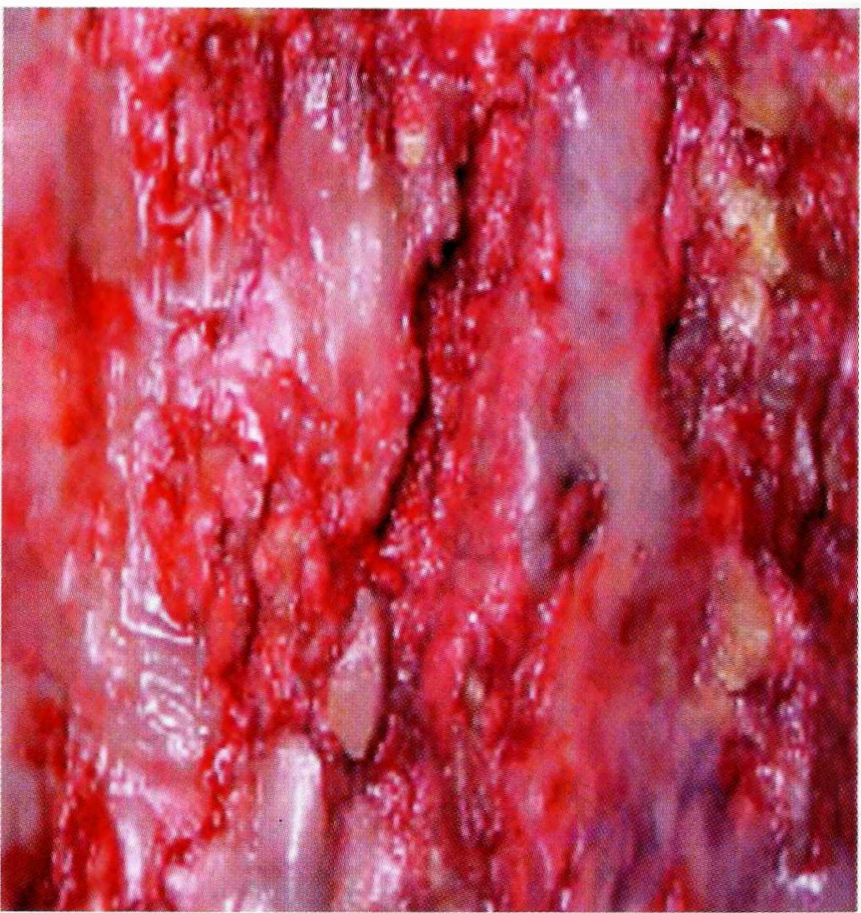

Figura 9. Placa complicada con técnica de Holman: zonas ulceradas, hemorrágicas, con presencia de calcificaciones.

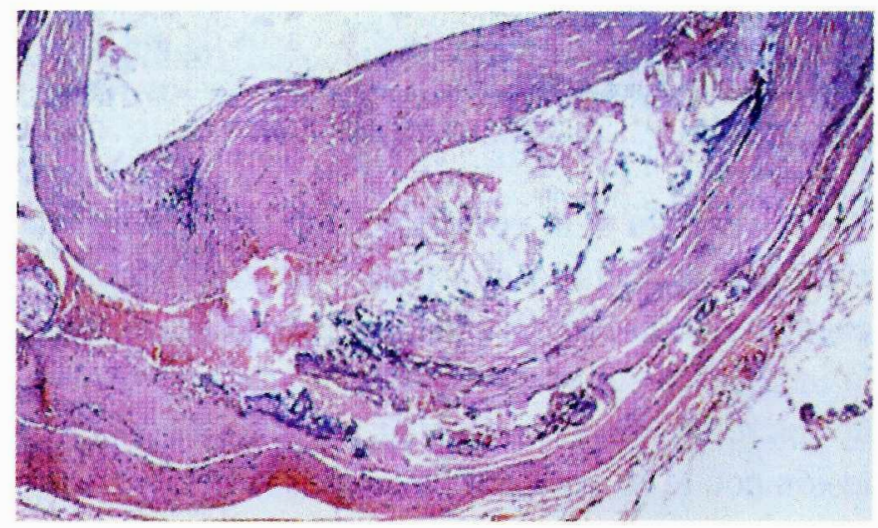

Figura 10. Coloración de $\mathrm{HE}$ con objetivo de $4 \mathrm{x}$. Existe pérdida de la estructura. 


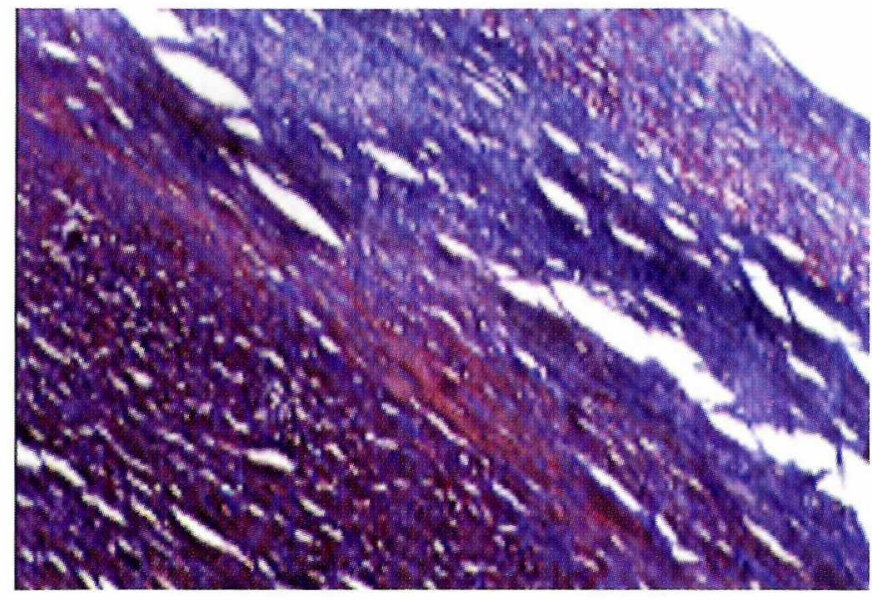

Figura 11. Coloración de tricrómico con objetivo de 10x. Se identifican pocas fibras colágenas y fibras musculares.

En la tabla 1 se observa cómo el comportamiento de la evolución de la estría adiposa con respecto a la edad, muestra su mayor incidencia entre los grupos etáreos comprendidos entre 15 a 35 años y la escasa probabilidad de presentarla en edades más avanzadas.

En la tabla 2 se puede establecer que la placa fibrosa con respecto a la edad en los pacientes sometidos al estudio evolucionó a medida que aumenta la edad. Como hallazgo importante se muestra la presencia de dicha lesión en un paciente muy joven, demostrando así los diferentes comportamientos que toman en cada individuo o población.

En la tabla 3 se analiza la placa complicada con respecto a la edad, lo que permitió establecer que la incidencia de esta lesión es mayor en personas de edad avanzada, exceptuando seis casos de pacientes entre los 35 y 44 años que se salen de los rangos normales.

En la tabla 4 la correlación edad vs índice de obstrucción registró una mayor lesión arterial en pacientes entre 40 y 60 años. Por debajo de esta edad la incidencia global de eventos fue menor afirmando los resultados de investigación anteriores que la lesión con el incremento de la edad.

En cada una de las tablas se registraron casos que se salen de los rangos normales lo cual nos hace pen-

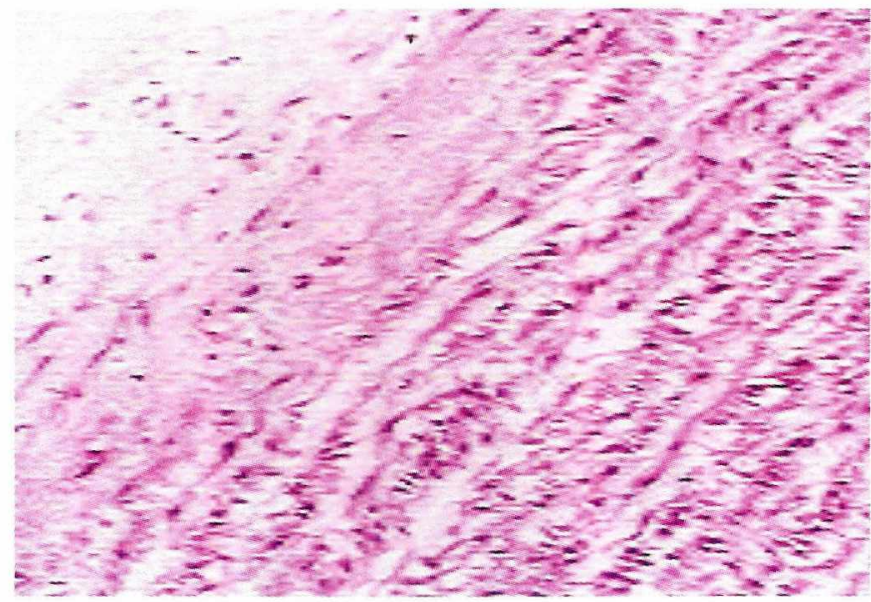

Figura 12. Coloración de HE con objetivo de 40x. Se observa con más detalle la neovascularización, así como la presencia de abundantes macrófagos y fibroblastos, con marcada inflamación.

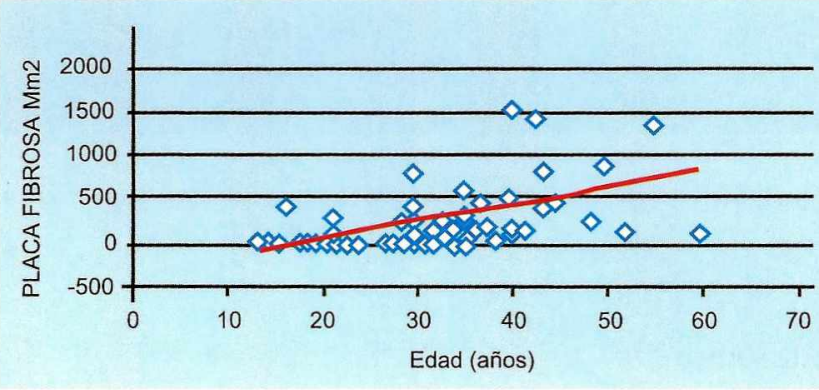

Tabla 1. Correlación edad vs. estría adiposa.

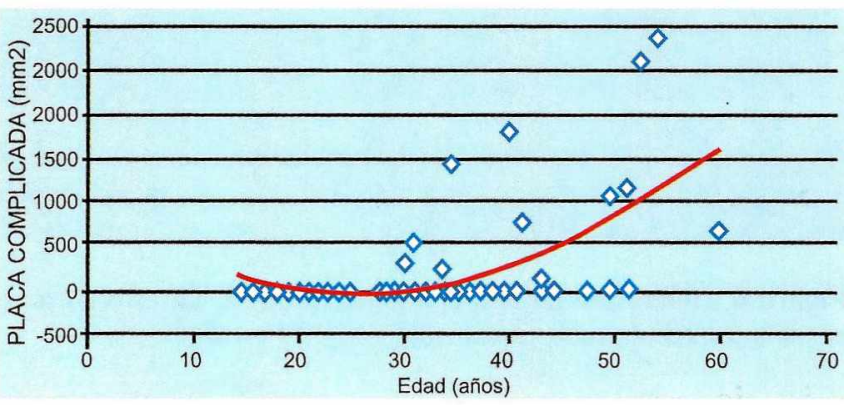

Tabla 2. Correlación edad vs. placa fibrosa.

sar que intervienen factores coadyuvantes exógenos o endógenos que influyen en cada individuo, para que incremente la evolución de la enfermedad.

\section{Discusión}

La distribución de las lesiones a pesar de la muestra tan pequeña, está, en términos generales, de acuer- 


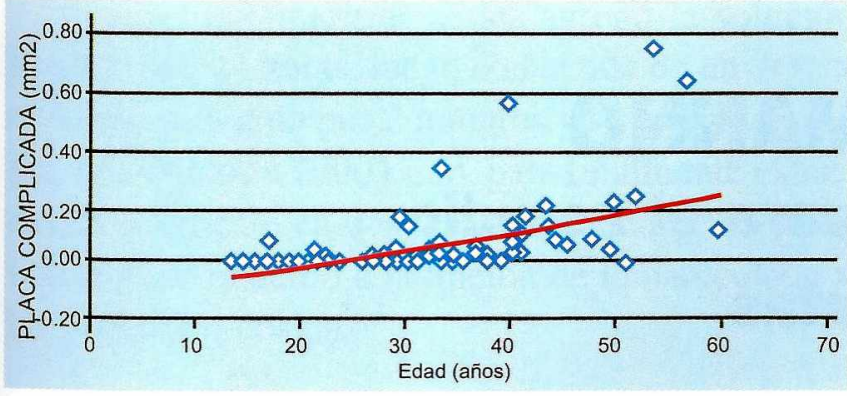

Tabla 3. Correlación edad vs. placa complicada.

do con lo descrito en investigaciones de otros países que muestran el desarrollo de la enfermedad ateroesclerótica como un proceso evolutivo de envejecimiento y deterioro de la arteria, que se puede ver influenciando por algún factor de riesgo (diabetes, obesidad, tabaquismo, hiperlipidemias), acelerando el proceso aterogénico y, por lo tanto, se puede presentar en edades más tempranas.

Los resultados obtenidos demuestran que esta técnica es una herramienta ágil y confiable para la identificación y medición de lesiones precursoras de la ateroesclerosis, y abre nuevas expectativas de investigación que permiten explorar el comportamiento de la enfermedad en otras arterias como carótidas, ilíacas y coronarias.

\section{Conclusiones}

Se concluye que el sistema aterométrico es válido para la identificación y medición de las lesiones precursoras de la $\mathrm{AE}$ y permite observar paso a paso la evolución de la enfermedad. Se adquirió gran experiencia y conocimiento teórico-práctico sobre el

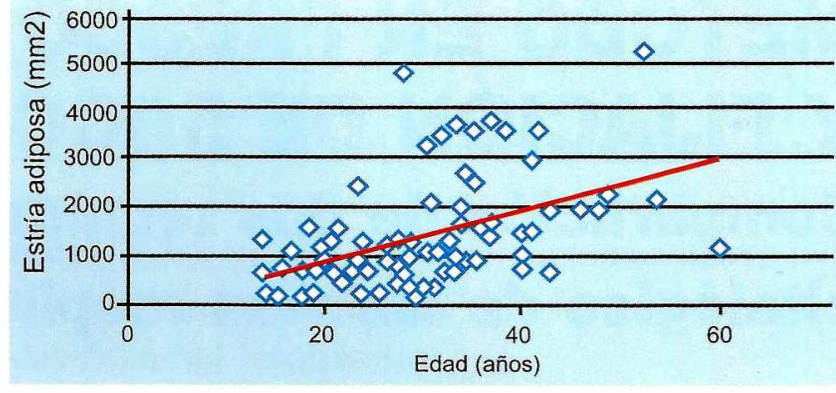

Tabla 4. Correlación edad-índice de obstrucción.

empleo del sistema aterométrico y el desarrollo de esta patología en pacientes jóvenes. Además, se da a conocer una nueva técnica para el estudio de lesiones ateromatosas, hasta el momento poco empleada en nuestro país.

Las lesiones macroscópicas evidenciadas en la técnica de Holman son compatibles con el diagnóstico histológico en microscopía óptica, ya que las coloraciones empleadas permitieron realizar una mejor diferenciación de los componentes celulares presentes en cada una de las lesiones precursoras de la $\mathrm{AE}$.

\section{Referencias}

1. Caraballe Blase A. Perlas de la cardiología. Mosby Doyma Libros; $\mathrm{p} 156$.

2. Fernández-Brito. Artículo del sistema aterométrico Cuba, 1980.

3. RMH Meminn RMH, Hutchings RJ. Atlas a color de anatomía Humana. Yeerra Book Medical Publishers I.N.C.; p 132. 4. Vélez H, Botero J, Rojas W. Cardiología fundamental de medicina. Carvajal S.A.; p 356.

5. García del Moral R. Laboratorio de anatomía patológica Mc Graw Hill 1993. capitulo 28; p 558. 